\title{
Confronting brane inflation with Planck and pre-Planck data
}

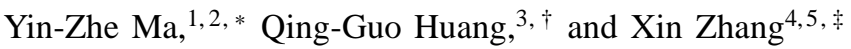 \\ ${ }^{1}$ Department of Physics and Astronomy, University of British Columbia, Vancouver, V6T 1Z1, BC, Canada \\ ${ }^{2}$ Canadian Institute for Theoretical Astrophysics, Toront, M5S 3H8, Ontario, Canada. \\ ${ }^{3}$ State Key Laboratory of Theoretical Physics, Institute of Theoretical Physics, Chinese Academy of Sciences, Beijing 100190, China \\ ${ }^{4}$ College of Sciences, Northeastern University, Shenyang 110004, China \\ ${ }^{5}$ Center for High Energy Physics, Peking University, Beijing 100080, China
}

\begin{abstract}
In this paper, we compare brane inflation models with the Planck data and the pre-Planck data (which combines WMAP, ACT, SPT, BAO and $H_{0}$ data). The Planck data prefer a spectral index less than unity at more than $5 \sigma$ confidence level, and a running of the spectral index at around $2 \sigma$ confidence level. We find that the KKLMMT model can survive at the level of $2 \sigma$ only if the parameter $\beta$ (the conformal coupling between the Hubble parameter and the inflaton) is less than $O\left(10^{-3}\right)$, which indicates a certain level of fine-tuning. The IR DBI model can provide a slightly larger negative running of spectral index and red tilt, but in order to be consistent with the non-Gaussianity constraints from Planck, its parameter also needs fine-tuning at some level.
\end{abstract}

\section{INTRODUCTION}

The ongoing astronomical observations, such as WMAP [1], Planck [2-4], SDSS [6], ACT [7] and SPT [8], have been measuring the cosmic microwave background (CMB) and large scale structure to an unprecedented precision. This provides an excellent opportunity to probe the physics in the early Universe with the underlying fundamental theories. One of the leading candidates of generating initial fluctuations in the early Universe is inflation [9, 10]. The inflation paradigm offers a compelling explanation for many puzzles in the standard hot big-bang cosmology, such as the flatness problem, homogeneity problem and horizon problem [9]. The accelerated expansion period in the early Universe provides a nearly scale-invariant primordial power spectrum which has already been supported by the measurements of CMB anisotropy [15, 7, 8]. In spite of its phenomenological success, inflation remains a paradigm rather than a fundamental theory, which in principle can be implemented by various models from different microscopic physical constructions [11]. The fact that it is easy to construct a wide variety of inflation models does not mean that any of them will turn out to be the true mechanism. Actually, effective field theory models of inflation should by definition be understood as valid only up to some energy scale that is low enough, and so the singularity problem and any "trans-Planckian" effects are out of the range of validity of the models [12, 13]. If one would like a UV completion to any effective field theory ideas, one might hope that the string theory would provide such a way. Undoubtedly, inflation can be successfully realized in a string context.

The string inflation model considered in this paper is the brane inflation scenario, proposed in [14, 15] originally, which offers a class of observational signatures. In this scenario, the inflation is driven by the potential between the parallel dynamic brane and antibrane [16-18], and the distance between the branes in the extra compactified dimensions plays the role

\footnotetext{
*Electronic address: mayinzhe@phas.ubc.ca

$\dagger$ Electronic address: huangqg@itp.ac.cn

‡Electronic address: zhangxin@mail.neu.edu.cn
}

of the inflaton field. This inflation scenario can be realized via two viable mechanisms, namely, the slow-roll and DiracBorn-Infeld (DBI) inflations [11].

The original brane inflation model is the slow-roll inflation model [14, 16-18] where branes and antibranes are slowly moving towards each other in a flat potential. The KKLMMT model [19] provides such an example. In this model, the antibrane is fixed at the bottom of a warped throat, while the brane is mobile and experiences a small attractive force towards the antibrane [19, 20]. When the brane and antibrane collide and annihilate, the inflation ends and the hot big-bang epoch is initiated. The annihilation of the brane and antibrane makes the universe settle down to the string vacuum state that describes our Universe [19, 20]. For extensive studies on the KKLMMT model and other types of slow-roll brane inflation models, see Refs. [11, 20-27].

Another inflationary mechanism is the DBI inflation. In this paradigm, the speed of the rolling brane is not determined by the shape of the potential but by the speed limit of the warped spacetime [28-32]. The warped internal spaces naturally arise in the extra dimensions due to the stabilized string compactification.

In order to test the inflationary paradigm and explore the dynamics of the internal space, we will scan the parameter spaces of these two types of inflation models subject only to the requirement that they provide enough $e$-folding number to solve the flatness, horizon and homogeneity problems. This is because solving the problems of standard cosmology is the basic motivation of the inflation paradigm and the most attractive feature of inflation models [11]. Then we will explore the observational signatures that are allowed by brane inflation dynamics and constrain the model parameters with the current observational CMB data. We will see that the current observational data are able to tighten up the parameter space of brane inflation to a great extent and the generic models need to be fine-tuned to match the current observations.

Recently the Planck team just released the results from the 2.7 full-sky surveys [2]. For the $\Lambda$ CDM model, Planck data combined with WMAP polarization data (hereafter Planck+ WP) show that the index of the power spectrum satisfies [3, 4]

$$
n_{s}=0.9603 \pm 0.0073 \quad(1 \sigma \mathrm{CL})
$$


at the pivot scale $k_{0}=0.05 \mathrm{Mpc}^{-1}$, which rules out the exact scale invariance $\left(n_{s}=1\right)$ at more than $5 \sigma$. If the running of spectral index $\alpha_{s}=d n_{s} / d \ln k$ is released as a free parameter, the spectral index becomes redder,

$$
n_{s}=0.9561 \pm 0.0080 \quad(1 \sigma \mathrm{CL}),
$$

while the running of the spectral index is not equal to zero at less than $2 \sigma \mathrm{CL}$,

$$
d n_{s} / d \ln k=-0.0134 \pm 0.0090 \quad(1 \sigma \mathrm{CL}) .
$$

For a comparison, in [36], we combined the WMAP 9-year data [1] with ACT data [7], SPT data [8], as well as BAO data [6, 33, 34] and $H_{0}$ data [35] (hereafter, we call this combined data set the "WMAP9+" data set), and we obtained a red spectral index of power spectrum at the pivot scale $k_{0}=0.002$ $\mathrm{Mpc}^{-1}$,

$$
n_{s}=0.961 \pm 0.007 \quad(1 \sigma \mathrm{CL}) .
$$

But if we let the running of the spectral index be $\alpha_{s}=$ $d n_{s} / d \ln k$ as a free parameter, the spectral index becomes

$$
n_{s}=1.018 \pm 0.027 \quad(1 \sigma \mathrm{CL}),
$$

and the running of the spectral index becomes

$$
\alpha_{s}=d n_{s} / d \ln k=-0.021 \pm 0.009(1 \sigma \mathrm{CL}) .
$$

In addition, the joint constraints on $r$ (tensor-to-scalar ratio) and $n_{s}$ already become a very sensitive tool to constrain inflation models. In [1], it is found that inflation models with power-law potential $\phi^{4}$ cannot provide a reasonable number of $e$-folds (between 50-60) in the restricted space of $r-n_{s}$ at around $2 \sigma$ level. Reference [36] pushes this limit further and shows that with the combination of WMAP9, ACT, SPT, BAO and $H_{0}$ data, the inflation potential with power law form $\phi^{p}$ can only survive if $p$ is in the range of $0.9-2.1$.

Besides the above conventional parameters that have been used to constrain inflation models, Planck data are also able to constrain the non-Gaussianity of primordial fluctuations. The Planck found that the local, equilateral and orthogonal types of non-Gaussianity are

$$
\begin{array}{cc}
f_{\mathrm{NL}}^{\text {local }}=2.7 \pm 5.8 & (1 \sigma \mathrm{CL}) \\
f_{\mathrm{NL}}^{\text {equil }}=-42 \pm 75 \quad(1 \sigma \mathrm{CL}) \\
f_{\mathrm{NL}}^{\text {orth }}=-25 \pm 39 \quad(1 \sigma \mathrm{CL}) .
\end{array}
$$

These place very tight constraints on the inflation model space.

Based on the WMAP 3-year and 5-year results, Refs. [23] and [20] investigated brane inflation models and showed that the KKLMMT model cannot fit WMAP+SDSS data at the level of one standard deviation and a fine-tuning (at least one part in a hundred) is needed at the level of two standard deviations. Since the CMB data have been dramatically improved over the past several years, it is meaningful to see how the status of brane inflation is affected by the arrival of the new CMB data, especially the Planck and WMAP9+ data. In this paper, we will have a close look at the constraints on the brane inflation models with the results from Planck [2-4] and pre-Planck surveys [1, 6-8].

This paper is organized as follows: In Sec. [II we discuss the relationship between the $e$-folding number of inflation and the pivot scale of observation. In Sec. III, we discuss a simple brane inflation model neglecting the problem of dynamic stabilization. This is the simplest brane inflation model one can achieve in the multidimensional spacetime. In Sec. IV] we focus on the KKLMMT model and compare the model predictions with observational data. In Sec. $\mathbf{V}$ we turn to the discussion of the infrared DBI inflation model and confront the model predictions with observational data. The conclusion is presented in the last section.

\section{NUMBER OF E-FOLDS}

Before we start to constrain any inflation model, we first address an important issue in the inflation model tests: how do we compare model predictions with observational data? Inflation models are actually models of different inflation potentials, where the amplitude and shape are the features of various models. In the community of inflation theorists, people use the amplitude of potential to characterize the energy scale of inflation and a set of "slow-roll" parameters to describe the shape of the potential. Usually the shape of the potential includes the "slope" and "curvature" parameters of the potential. For a given potential, the slow-roll parameters can be expressed in terms of the number of $e$-folds $\left(N_{e}\right)$ which characterizes the duration of inflation.

On the other hand, observations from the CMB provide constraints on the amplitude and shape of the primordial power spectrum. But since the power spectrum itself is a $k$-dependent quantity, the measured amplitude $\left(\Delta_{\mathcal{R}}^{2}\right)$, tilt $\left(n_{s}\right)$, tensor-to-scalar ratio $(r)$ and running of spectral index $\left(d n_{s} / d \ln k\right)$ are referred to a particular "pivot scale". This indicates that for a given data set, if the pivot scale is switched to a different value, the constraints on the $\left(\Delta_{\mathcal{R}}^{2}\left(k_{0}\right), n_{s}\left(k_{0}\right)\right.$, $d n_{s} / d \ln k$ ) can be slightly different. Therefore, to really compare model predictions with observational data, we need to associate the number of $e$-folds with the pivot scale of observation. Our main goal in this section is to obtain a relationship between the number of $e$-folds $N_{e}$ and its corresponding comoving scale $k$.

Once inflation happened, different scales (different $k$-modes) stretched out of the Hubble radius at different time. After inflation, the Universe experienced a short period of reheating, and then entered into radiation, matter and dark energy dominated eras. The number of $e$-folds is related to the processes of subsequent evolution because both the inflation and subsequent evolutionary processes contribute to the total expansion factor of the Universe (see Fig. 1 in [37]). We can therefore write [37]

$$
\frac{k}{a_{0} H_{0}}=\frac{a_{\mathrm{k}} H_{\mathrm{k}}}{a_{0} H_{0}}=\left(\frac{a_{\mathrm{k}}}{a_{\mathrm{e}}}\right)\left(\frac{a_{\mathrm{e}}}{a_{\mathrm{reh}}}\right)\left(\frac{a_{\mathrm{reh}}}{a_{\mathrm{eq}}}\right)\left(\frac{H_{\mathrm{k}}}{H_{\mathrm{eq}}}\right)\left(\frac{a_{\mathrm{eq}} H_{\mathrm{eq}}}{a_{0} H_{0}}\right),
$$

where we used the subscripts " $k$, e, reh, eq, 0" to represent 
the horizon exit, end of inflation, reheating epoch, matterradiation equality epoch and present time. Number of $e$ folds between horizon exit and the end of inflation is $N_{e}(k)=$ $\ln \left(a_{e} / a_{\mathrm{k}}\right)$. By assuming the equation of state during the reheating era being $w(w=P / \rho)$, one can reach the following equation (see also [37, 41]),

$$
\begin{aligned}
N_{e}(k)= & -\ln \left(\frac{k}{a_{0} H_{0}}\right)+\ln \left(\sqrt{\frac{V_{\mathrm{k}}}{3 M_{\mathrm{pl}}^{2}}} \frac{1}{H_{\mathrm{eq}}}\right)+\ln \left(219 \Omega_{\mathrm{m}} h\right) \\
& -\frac{1}{3(1+w)} \ln \left(\frac{\rho_{\mathrm{e}}}{\rho_{\mathrm{reh}}}\right)-\frac{1}{4} \ln \left(\frac{\rho_{\mathrm{reh}}}{\rho_{\mathrm{eq}}}\right),
\end{aligned}
$$

where $V_{\mathrm{k}}$ is the energy scale of inflation at horizon exit, and $M_{\mathrm{pl}} \equiv 1 /(8 \pi G) \simeq 2.4 \times 10^{18} \mathrm{GeV}$ is the reduced Planck mass. By defining the ratio of the energy densities between at the reheating and at the end of inflation as $x \equiv \rho_{\mathrm{reh}} / \rho_{\mathrm{e}}$, and regarding $\rho_{\mathrm{e}}=V_{\mathrm{k}}$ ("slow-roll" approximation), we can rewrite Eq. (9) as

$$
\begin{aligned}
N_{e}(k)= & -\ln \left(\frac{k}{a_{0} H_{0}}\right)+\ln \left(\sqrt{\frac{V_{\mathrm{k}}}{3 M_{\mathrm{pl}}^{2}}} \frac{1}{H_{\mathrm{eq}}}\right)+\ln \left(219 \Omega_{\mathrm{m}} h\right) \\
& +\left(\frac{1}{3(1+w)}-\frac{1}{4}\right) \ln x+\frac{1}{4} \ln \left(\frac{\rho_{\mathrm{eq}}}{V_{\mathrm{k}}}\right) .
\end{aligned}
$$

To further simplify this equation, we use the requirement that the primordial perturbations have to produce the observed level of fluctuations $\left(P_{s}\left(k_{0}\right) \simeq 2.43 \times 10^{-9}\right)$, i.e.,

$$
P_{s}=\frac{V_{\mathrm{k}} / M_{\mathrm{pl}}^{4}}{24 \pi^{2} \epsilon_{v}}, \text { where } \epsilon_{v}=\frac{M_{\mathrm{pl}}^{2}}{2}\left(\frac{V^{\prime}}{V}\right)^{2} .
$$

Substituting known quantities, Eq. (10) can be greatly simplified as

$$
\begin{aligned}
N_{e}(k)= & -\ln \left(\frac{k}{2.33 \times 10^{-4} \mathrm{Mpc}^{-1}}\right)+63.3+\frac{1}{4} \epsilon_{v} \\
& +\left(\frac{1}{3(1+w)}-\frac{1}{4}\right) \ln x .
\end{aligned}
$$

For a particular mode $k$, its corresponding $N_{e}(k)$ relies on the equation of state $w$ and energy scale of reheating $\rho_{\text {reh }}$. Since the standard picture tells that vacuum is decayed into standard particles, $\rho_{\text {reh }}$ is always less than or equal to potential energy scale $V_{k}$, i.e. $x \leq 1$, thus $\ln x$ is always a negative value. Therefore, if $w \rightarrow 0$ (close to a "matter-dominated phase"), the fourth term of Eq. (12) becomes $(1 / 12) \ln x$, which gives a minimal number of $e$-folds. This means that if the equation of state is close to zero, the shape of the potential $\left(\sim \phi^{2}\right)$ can keep inflaton oscillating for a fairly long period of time while the Universe is expanding, therefore we need less number of $e$-folds to produce an observable scale of the Universe. On the other hand, if the equation of state during the reheating era is $w \simeq 1 / 3$, or the reheating is instantaneous $\left(\rho_{\text {reh }}=V_{\mathrm{k}}\right.$, i.e., $\ln x=0$ ), the fourth term vanishes, which gives the maximum number of $e$-folds $\left(\sim \phi^{4}\right)$. Since there is a great uncertainty of what energy scale reheating really happened, in the following discussion we stick to the case of instantaneous reheating, so that the number of $e$-folds becomes

$$
N_{e}(k)=-\ln \left(\frac{k}{2.33 \times 10^{-4} \mathrm{Mpc}^{-1}}\right)+63.3+\frac{1}{4} \epsilon_{v} .
$$

For joint $W M A P 9+\mathrm{SPT}+\mathrm{ACT}+\mathrm{BAO}+H_{0}$ data $\left(k_{0}=\right.$ $\left.0.002 \mathrm{Mpc}^{-1}\right)$ and Planck+WP data $\left(k_{0}=0.05 \mathrm{Mpc}^{-1}\right)$, the corresponding numbers of $e$-folds are

$$
\begin{gathered}
N\left(k_{0}\right)=61.2+\frac{1}{4} \ln \epsilon \quad(\text { for WMAP9+), } \\
N\left(k_{0}\right)=58.2+\frac{1}{4} \ln \epsilon \quad(\text { for Planck }+\mathrm{WP}) .
\end{gathered}
$$

Typically observational predictions of slow-roll parameters (e.g. $\epsilon_{v}$ ) depend on $N_{e}$, so both sides of Eq. (14) contain $N_{e}$ which could be solved simultaneously. In practice, the deviation of $N_{e}$ from the typical value 60 is always small, so one can solve Eq. (14) iteratively by assuming a particular $N_{e}$ and use it to calculate the potential properties, then use these to recalculate $N_{e}$, and so on. In fact, one iteration easily suffices to give sufficient accuracy of $N_{e}$. We will illustrate this in the following sections.

\section{A TOY MODEL}

\section{A. Model predictions}

To begin with, we consider a toy model of brane inflation; actually, this is a prototype of the brane inflation: a pair of $D p$ and $\bar{D} p$-branes $(p \geq 3)$ fill the four large dimensions and are separated from each other in the extra six dimensions that are compactified. Note that this model is not a realistic working model because it does not take into account the warped space-time and moduli stabilization. However, such a prototype provides us with a warm-up exercise for comparing models with CMB observations. In this model, the inflaton potential is given by [17, 20, 23]

$$
V=V_{0}\left(1-\frac{\mu^{n}}{\phi^{n}}\right)
$$

where $V_{0}$ is an effective cosmological constant on the brane and the second term in Eq. (15) is the attractive force between the branes. The parameter $n$ has to satisfy $n \leq 4$ because the transverse dimension has to be less or equal to 6 . The $e$-folding number $N_{e}$ at the horizon exit before the end of inflation is related to the field value as [20, 23]

$$
\phi_{N}=\left[N_{e} M_{\mathrm{pl}}^{2} \mu^{n} n(n+2)\right]^{1 /(n+2)} .
$$

The slow-roll parameters have been calculated as [17, 20, 23]

$$
\begin{aligned}
\epsilon_{v} & =\frac{M_{\mathrm{pl}}^{2}}{2}\left(\frac{V^{\prime}}{V}\right)^{2} \\
& =\frac{n^{2}}{2(n(n+2))^{\frac{2(n+1)}{n+2}}}\left(\frac{\mu}{M_{\mathrm{pl}}}\right)^{\frac{2 n}{n+2}} N_{e}^{-\frac{2(n+1)}{n+2}},
\end{aligned}
$$




$$
\begin{aligned}
& \eta_{v}=M_{\mathrm{pl}}^{2} \frac{V^{\prime \prime}}{V}=-\frac{n+1}{n+2} \frac{1}{N_{e}}, \\
& \xi_{v}=M_{\mathrm{pl}}^{4} \frac{V^{\prime} V^{\prime \prime \prime}}{V^{2}}=\frac{n+1}{n+2} \frac{1}{N_{e}^{2}} .
\end{aligned}
$$

The observational quantities, $n_{s}, r$, and $\alpha_{s}$ (spectral index, tensor-to-scalar ratio, and running of spectral index), can be expressed as the combination of slow-roll parameters

$$
\begin{aligned}
n_{s} & =1+2 \eta_{v}-6 \epsilon_{v}, \\
r & =16 \epsilon_{v}, \\
\alpha_{s} & =-24 \epsilon_{v}^{2}+16 \epsilon_{v} \eta_{v}-2 \xi_{v} .
\end{aligned}
$$

These are the observables that we will compare with observational results.

\section{B. Constraints from Planck and pre-Planck data}

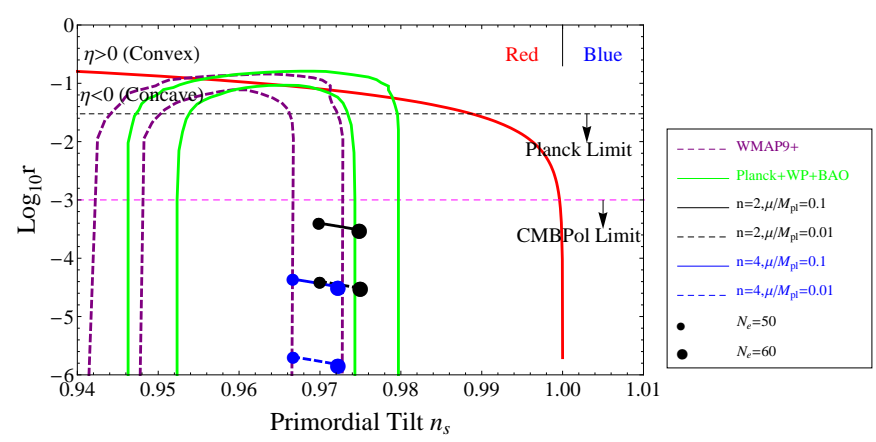

FIG. 1: The $r-n_{s}$ plot for theoretical models, current observational constraints and predicted limits from Planck polarization maps and CMBPol. For model predictions: the red curve across the whole diagram is the divided line for $\eta=0$, on either side the potential has different curvatures as marked onto the plot. The black and blue lines are the predictions for the $n=2$ and $n=4$ models with $\mu / M_{\mathrm{pl}}=0.1$ (solid line) and 0.01 (dashed line). The small and big dots correspond to $N_{e}=50$ and 60, respectively. We also mark the red tilt and blue tilt on the top of the diagram. For the observational results: the purple dashed contours are the joint constraints from $W M A P 9+\mathrm{SPT}+\mathrm{ACT}+\mathrm{BAO}+H_{0}$ ("WMAP9+") and the green solid contours are the joint constraints from Planck+WP+BAO. The two horizontal dashed lines are the predicted observational limits of tensor-to-scalar ratio $r$ from Planck polarization map $(r \lesssim 0.03)$ [38] and CMBPol $(r \lesssim 0.001)$ [39, 40]; note that the two lines are not from actual data, but are based on the predictions of future data.

In Fig. 1, we plot the theoretical prediction of $r$ (in terms of $\left.\log _{10} r\right)$ and $n_{s}$. The black and blue lines are the predictions for $n=2$ and $n=4$ models with $\mu / M_{\mathrm{pl}}$ values being 0.1 and 0.01 . The range between small and big dots corresponds to the number of $e$-folds within [50,60]. The red line across the diagram is the boundary line between the convex potential $\left(\eta_{v}>0\right)$ and concave potential $\left(\eta_{v}<0\right)$. We also plot the $1 \sigma$ and $2 \sigma$ constraints on $r$ and $n_{s}$ from WMAP9+ data and
Planck+WP+BAO (hereafter Planck+) data. From the plot, one can see that WMAP9+ prefers a slightly lower $n_{s}$ comparing with Planck+ data. In addition, most of the contour regions locate within $\eta_{v}<0$ region, indicating strong evidence of concave potential. The models with $n=2$ and $n=4$ lying within the contours suggests that the model prediction is consistent with the current constraints. We also plot the predicted detection limits of $r$ from Planck polarization experiment [38] and CMBPol [39]; note that these two limits are not from actual data, but are based on the predictions of future data. One can see that even if $\mu / M_{\mathrm{pl}}$ is of order 0.1 , the model prediction is still much lower than the CMBPol detection limit. Only if $\mu / M_{\mathrm{pl}}>0.3$, could the CMBPol be able to detect the tensor mode in this model.

In Fig. 2 we plot the predicted $\alpha_{s}-n_{s}$ relation for the brane inflation model with the constraint results from WMAP9+ and Planck+ data. The purple contours on the left panel is the joint constraints on $\alpha_{s}-n_{s}$ from the WMAP9+ data set with the pivot scale $k_{0}=0.002 \mathrm{Mpc}^{-1}$. Therefore we use Eq. (14) to determine the number of $e$-folds: we substitute a fiducial number of $e$-folds $N_{\text {fid }}=60$ into Eq. (17) and obtain an estimate of $\epsilon$, then substitute it into Eq. (14) to obtain the corresponding number of $e$-folds for this model. We test that one iteration is enough for determine the specific $N_{e}$. Then with Eqs. (17)(20) we plot the $\alpha_{s}-n_{s}$ prediction with variation of the parameter $\mu$. The red line is for the $n=2$ model and the blue line is for the $n=4$ model. The two lines are pretty close to each other, and they are all outside $1 \sigma$ confidence level (CL) but some range is within $2 \sigma \mathrm{CL}$. We then figure out which values of $\mu$ can match the results inside the $2 \sigma$. We give a couple of trials and find that, for the $n=2 \operatorname{model} \mu / M_{\mathrm{pl}}$ needs to be between $10^{-48}$ and unity, and for the $n=4$ model this range is $\left[10^{-30}, 1\right]$. On the right panel of Fig. 2, we use the constraints from Planck+WP+BAO to compare with the theoretical predictions. The results are similar to the left panel, except that the range of $\mu / M_{\mathrm{pl}}$ is shorten to be $\left[10^{-46}, 1\right]$ for the $n=2$ model, and $\left[10^{-29}, 1\right]$ for the $n=4$ model. In a word, the prototype of brane inflation with potential form (15) is consistent with the observational constraints on $\alpha_{s}$ and $n_{s}$.

Then let us see what this implies for the energy scale of inflation in this model. The amplitude of the scalar perturbations is [21, 23, 41]

$$
\Delta_{\mathcal{R}}^{2}=\frac{V}{M_{\mathrm{pl}}^{4}} \frac{1}{24 \pi^{2} \epsilon_{v}}
$$

which is constrained to be $\sim 2.2 \times 10^{-9}$ by the Planck data [2]. We substitute $\epsilon_{v}$ [Eq. (17)] into Eq. (21), and thus we obtain a relationship between the amplitude of inflation and the parameter $\mu$,

$$
\frac{V^{\frac{1}{4}}}{M_{\mathrm{pl}}}=\left(24 \pi^{2} \frac{n^{2}}{2(n(n+2))^{\frac{2(n+1)}{n+2}}}\left(\frac{\mu}{M_{\mathrm{pl}}}\right)^{\frac{2 n}{n+2}} N_{e}^{-\frac{2(n+1)}{n+2}}\right)^{\frac{1}{4}}
$$

Then from our estimation of $\mu$ we can find that the amplitude of inflation is in the range $\left[2.7 \times 10^{4}, 8.4 \times 10^{15}\right] \mathrm{GeV}$ for the $n=2$ model and $\left[1.3 \times 10^{6}, 5.9 \times 10^{15}\right] \mathrm{GeV}$ for the $n=4$ model. These are all reasonable ranges for $V$, because it needs 

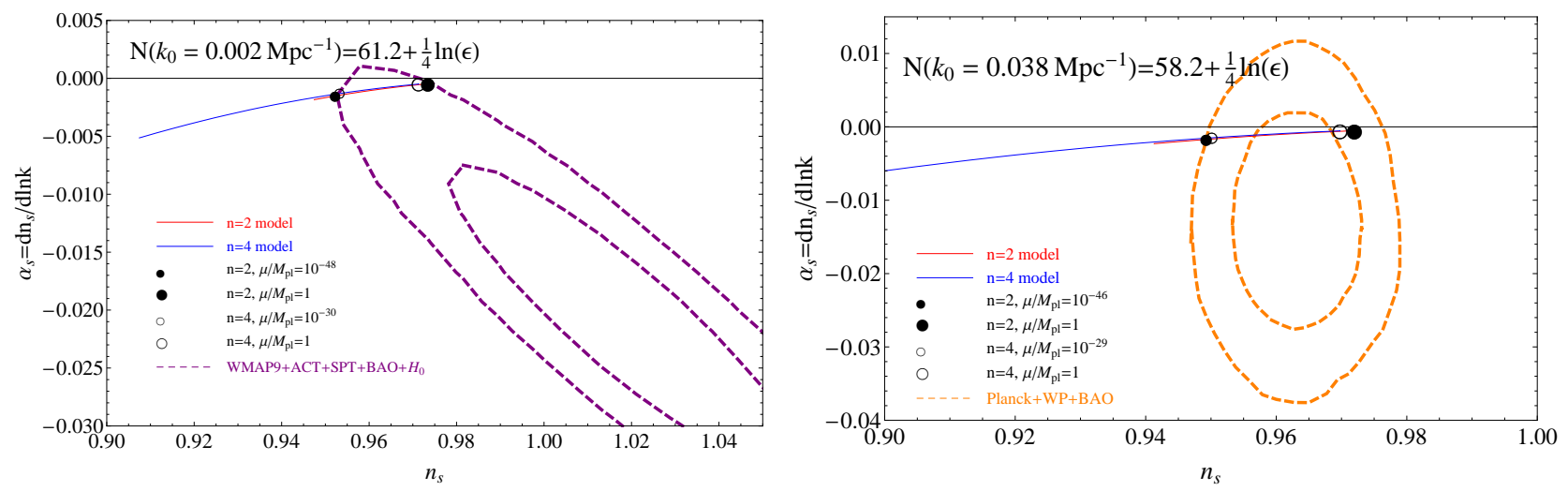

FIG. 2: Comparing the prediction of the prototype of brane inflation with the observational constraints on the $d n_{s} / d \ln k-n_{s}$ plane. $L e f t-$ Comparing with the joint constraints from combination of WMAP 9-year data, ACT, SPT, BAO and $H_{0}$ data at the pivot scale $k_{0}=0.002$ $\mathrm{Mpc}^{-1}$. Right-Comparing with the joint constraints from Planck+WP+BAO data at the pivot scale $k_{0}=0.038 \mathrm{Mpc}^{-1}$. In both panels, the number of $e$-folds of model predictions matches the pivot scale of the constrained contours. See text for more details of the theoretical predictions of the model.

to be lower than $10^{16} \mathrm{GeV}$ so that we do not detect any tensor mode yet, and greater than the particle physics energy scale $10^{3} \mathrm{GeV}$ since the inflaton is not detected in LHC.

\section{KKLMMT MODEL}

The prototype of the brane inflation model discussed above is not a realistic model, because the distance between the brane and the antibrane would be larger than the size of the extra-dimensional space if the inflaton is slowly rolling in this scenario [20, 23]. It indicates that this model is not really reliable from the viewpoint of theory itself. The first more realistic brane inflation model which considers the effect of warped spacetime on inflaton potential is the so-called KKLMMT model [19], whose predictions are directly calculable and can be directly compared to observations. Note that, strictly speaking, the KKLMMT model is only a braneinflation-inspired model rather than a scenario with all elements of the potential computed precisely; for more complicated versions of brane inflation, see [26, 27].

\section{A. Model predictions}

The KKLMMT model is derived from the type IIB string theory. In the model, the spacetime contains highly warped compactifications, and all moduli stabilized by the combination of fluxes and nonperturbative effects [19, 20]. Once a small number of $\overline{\mathrm{D}} 3$-branes are added, the vacuum can be successfully lifted to de Sitter state. Furthermore, one can add an extra pair of D3-brane and $\overline{\mathrm{D}} 3$-brane in a warped throat with the D3-brane moving towards the $\overline{\mathrm{D}} 3$-brane that is located at the bottom of the throat. When the D3 moves towards the $\overline{\mathrm{D}} 3$, inflation takes place; therefore, the scenario of brane inflation can be achieved in this model. The warped throat successfully guarantees a flat potential, which solves the " $\eta$ problem" in the brane inflation.

Let us start with the inner space of the Calabi-Yau manifold, where the geometry is highly warped and its spacetime can be approximate $A d S_{5} \times X_{5}$ form. The $A d S_{5}$ metric in Poincaré coordinates has the form [20-22]

$$
d s^{2}=h^{-\frac{1}{2}}(r)\left(-d t^{2}+a(t)^{2} d \vec{x}^{2}\right)+h^{\frac{1}{2}}(r) d s_{6}^{2},
$$

where $h(r)$ is the warp factor,

$$
h(r)=\frac{R^{4}}{r^{4}},
$$

where we express the radius of curvature of the $A d S_{5}$ throat as $R$. The potential within the warped throat is

$$
V(\phi)=\frac{1}{2} \beta H^{2} \phi^{2}+2 T_{3} h^{4}\left(1-\frac{\mu^{4}}{\phi^{4}}\right),
$$

which basically constitutes three terms. The first term is the Kähler potential term which arises from interactions of superpotentials [21] where $H$ is the Hubble parameter and $\beta$ describes the coupling between inflaton $\phi$ (position of D3 brane) and space expansion. In general, the value of $\beta$ depends on $\phi$ value because the conformal coupling depends on the position of the D3 brane, but we expect that $\beta$ to stay more or less constant in each throat, so approximately $\beta \simeq$ const here [21]. Generically $\beta \sim 1$, but for KKLMMT type of slow-roll model, $|\beta|$ is much less than unity. The second term $\left(2 T_{3} h^{4}\right)$ is the effective cosmological constant in the brane [21]. This is the term that drives the accelerated expansion of the Universe. The last term (with minus sign) provides the Coulomb-like attractive potential between the D3-brane and the D3-brane, making the two branes eventually collide. Note that $T_{3}$ is the D3-brane tension and it is related to $\mu$ through $\mu^{4}=\frac{27}{32 \pi^{2}} T_{3} h^{4}$. We then have

$$
V(\phi)=\frac{1}{2} \beta H^{2} \phi^{2}+\frac{64 \pi^{2} \mu^{4}}{27}\left(1-\frac{\mu^{4}}{\phi^{4}}\right) .
$$


Under the slow roll approximation, the Friedmann equation becomes

$$
3 M_{\mathrm{pl}}^{2} H^{2} \simeq V(\phi) \simeq V_{0}=\frac{64 \pi^{2} \mu^{4}}{27} ;
$$

therefore, $\mu$ also represents the energy scale of inflation.

Given the potential, it becomes a standard calculation to obtain the field value at the onset of inflation and the set of slow-roll parameters. Following [20, 21, 23], we have

$$
\phi_{N}^{6}=24 M_{\mathrm{p} 1}^{2} \mu^{4} m(\beta)
$$

where

$$
m(\beta)=\frac{e^{2 \beta N}(1+2 \beta)-\left(1+\frac{1}{3} \beta\right)}{2 \beta\left(1+\frac{1}{3} \beta\right)} .
$$

Therefore, the slow-roll parameters in the KKLMMT model are

$$
\begin{gathered}
\epsilon_{v}=\frac{1}{18}\left(\frac{\phi_{N}}{M_{\mathrm{pl}}}\right)^{2}\left[\beta+\frac{1}{2 m(\beta)}\right]^{2}, \\
\eta_{v}=\frac{\beta}{3}-\frac{5}{6} \frac{1}{m(\beta)}, \\
\xi_{v}=\frac{5}{3} \frac{1}{m(\beta)}\left[\beta+\frac{1}{2 m(\beta)}\right] .
\end{gathered}
$$

Now we need to use the observed CMB fluctuations to fix the amplitude of the scalar perturbations. Similar to the calculation we did in Sec. IIIB, we obtain

$$
\Delta_{\mathcal{R}}^{2} \simeq \frac{V}{M_{\mathrm{pl}}^{4}} \frac{1}{24 \pi^{2} \epsilon_{v}}=\frac{2}{27 m(\beta)}\left(\beta+\frac{1}{2 m(\beta)}\right)^{-2}\left(\frac{\phi_{N}}{M_{\mathrm{pl}}}\right)^{4},
$$

and thus we have

$$
\epsilon_{v}=\frac{1}{48}\left(\frac{3}{2}\right)^{\frac{1}{2}}\left(\Delta_{\mathcal{R}}^{2}\right)^{\frac{1}{2}} m(\beta)^{-\frac{5}{2}}(1+2 \beta m(\beta))^{3} .
$$

The Planck data give the amplitude of the primordial scalar power spectrum as $\Delta_{\mathcal{R}}^{2} \simeq 2.2 \times 10^{-9}$ for $N \sim 50$ [3]. Therefore, all of the slow-roll parameters in the KKLMMT model [Eqs. (30)-(32)] are related to the parameter $\beta$ and the number of $e$-folds $N_{e}$. Following Eq. (20), we will use parameters $n_{s}, \alpha_{s}$ and $r$ to figure out the best $\beta$ value given the current observational data.

\section{B. Constraints from observational data}

In Fig. 3, we plot the $r-n_{s}$ diagram similar to the structure of Fig. 11 Instead, here it is the KKLMMT model. The black solid and black dashed lines represent the trajectories for $N_{e}=50$ and 60, respectively. Different colors of empty and filled circles mark the point where the model takes different $\beta$ values. One can see how the $\beta$ parameter controls the shape of the potential. If it is greater than 0.03 , the potential turns to be convex which is not preferred by current observational data. Actually, the problem for $\beta>0.01$ is that it provides a blue tilt which has already been ruled out by Planck+WP+BAO at more than $5 \sigma \mathrm{CL}$. In order for the model to pass this test, $\beta$ value has to be much smaller than $10^{-3}$. In fact, since the current Planck data prefer the $n_{s}$ value around 0.96 (green contours), the models with $\beta<10^{-3}$ are just about to survive since they offer the spectral index to be 0.96 but not smaller than 0.95 (see Fig. 4 as well). This means that as long as the CMB data prefer $n_{s}$ to be around 0.96 , this model can always pass this test and survive. Nevertheless, the parameter needs to be highly fine-tuned. Finally, similar to Fig. 11 one can see that the tensor mode predicted by the KKLMMT model is really undetectably small since it is several orders of magnitude lower than the Planck polarization [38] and CMBPol limits [20, 39].

In Fig. 4 4 we show the comparison of the observational constraints and the model predictions on the $\alpha_{s}-n_{s}$ plane. One can see that Planck+WP+BAO prefers a slightly negative running with a very red power tilt. The tilt of the power spectrum at more than $5 \sigma$ deviates from unity (the Harrison-Zel'dovich spectrum), while the running is at less than $2 \sigma$ away from zero. On the other hand, if $\alpha_{s}$ is released as a free parameter, the WMAP9+ data set cannot tighten up $n_{s}$ to be less than unity. The purple contours stretch from a small negative running $(\sim-0.01)$ with red tilt $(\sim 0.96)$ out to a large negative running $(\sim-0.04)$ with blue tilt $(\sim 1.05)$ region. However, constraints from these two different data sets overlap at the small negative running and red tilt region, indicating that this is the preferable region for both data sets. In addition, we plot the model predictions for different $\beta$ values, and we mark the region of model predictions in between $N_{e}=50$ and 60 in order to have a direct vision of whether this "physically plausible" region falls in the observational constraint contours. One can also see that the KKLMMT model cannot produce a red tilt and suitable level of negative running unless $\beta \leq 10^{-3}$ at $2 \sigma \mathrm{CL}$. The model with $\beta=0.01$ cannot fit the $2 \sigma$ joint constraints in either case. This is actually an order of magnitude tighter than the previous upper limit of $\beta$ from WMAP 5-year data [20] $(\beta<0.01$ at $2 \sigma \mathrm{CL})$, and also much tighter than the combined constraints $\left(\beta<6 \times 10^{-3}\right)$ from WMAP3+SDSS [23].

\section{IR DBI MODEL}

\section{A. Model predictions}

In this section, we discuss another important type of brane inflation model, namely, the infrared Dirac-Born-Infeld model (IR DBI model). The difference between this model and the KKLMMT model is that the rolling velocity of the brane is not determined by the shape of the potential but by the speed limit of the warped spacetime [11]. Such a warped spacetime can always emerge in the inner space of compactified Calabi-Yau manifold. 


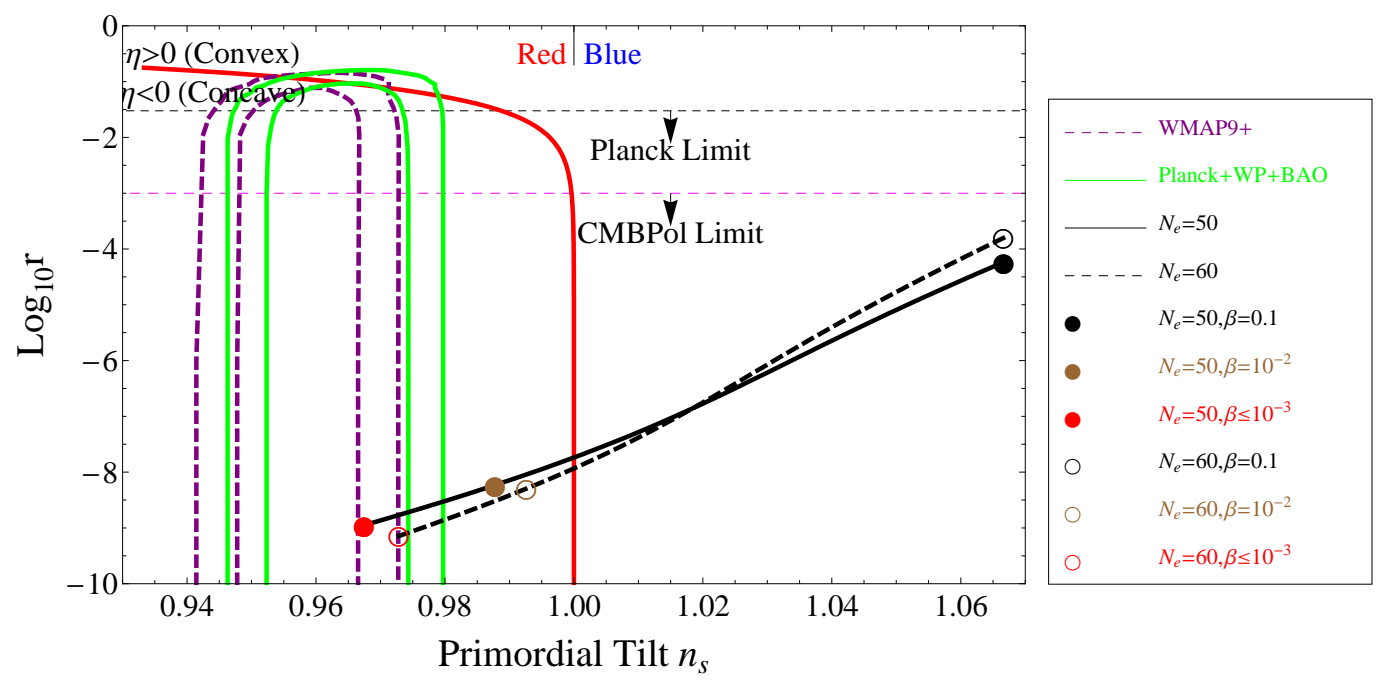

FIG. 3: Similar plot as Fig. 1 but for the KKLMMT model. The black solid and black dashed lines represent the trajectories for $N_{e}=50$ and 60, respectively. The empty and filled circles mark the points where the model takes $\beta=0.1$ (black), 0.01 (brown) and $\leq 0.001$ (red), respectively.

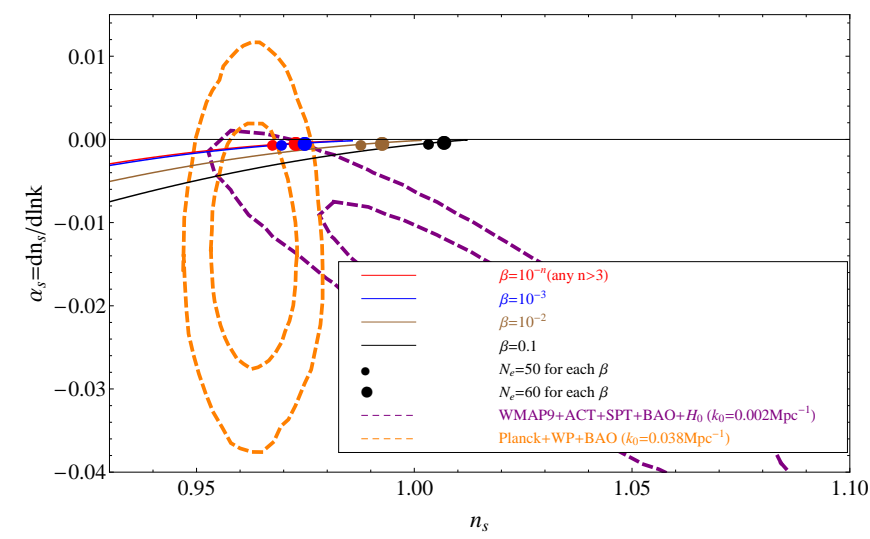

FIG. 4: Comparison of the joint observational constraints with the KKLMMT model predictions on the $\alpha_{s}-n_{s}$ plane. The purple and orange contours are the results from WMAP9+ and Planck+WP+BAO. The black, brown and blue lines are for models with $\beta=0.1,10^{-2}$, and $10^{-3}$, respectively. The red line is for any model with $\beta<10^{-3}$. The small and big color dots denote $N_{e}=50$ and 60, respectively.

Phenomenologically, the inflaton in IR DBI model can be driven by the kinetic term, where the inflaton is not slowly rolling at all. Therefore, the sound speed of inflaton in such a model could be less than unity, providing a large tilt in the tensor power spectrum (remember $n_{t}=-r /\left(8 c_{s}\right)$ [20, 42]). Observation on large scale temperature and polarization can be used to pin down the uncertainty of the soundspeed. In addition, as shown in previous analyses [11], there are a lot of parameters that describe the structure of internal space, and we will show that some of them may be pinned down by the CMB observations (see also [4] for more detailed discussions on constraints from non-Gaussianity).

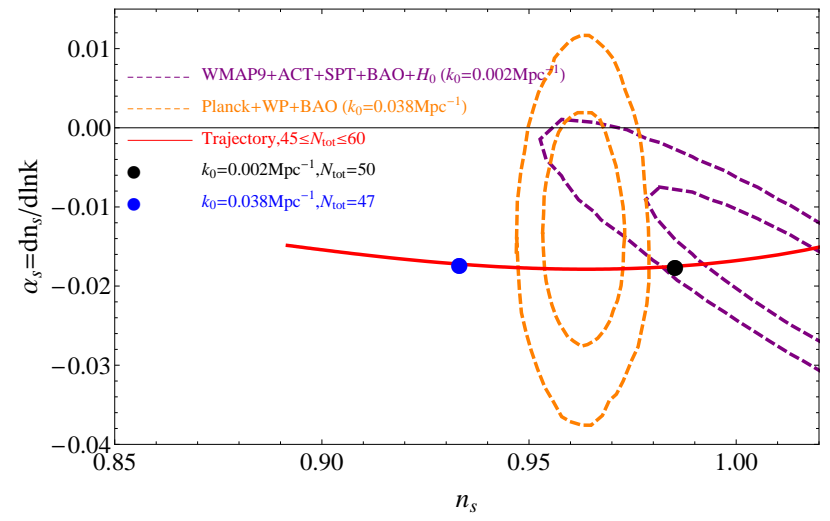

FIG. 5: Comparison of the constraints on $\alpha_{s}-n_{s}$ with the IR DBI model predictions. The red line is the trajectory of the number of $e$ folds in range of 45-60. The blue and black dots corresponds to the particular numbers of $e$-folds for the pivot scales $k_{0}=0.038 \mathrm{Mpc}^{-1}$ and $k_{0}=0.002 \mathrm{Mpc}^{-1}$.

In the DBI inflation, the action takes the form

$$
P(\phi, X)=-f(\phi)^{-1} \sqrt{1-2 f(\phi) X}+f(\phi)^{-1}-V(\phi),
$$

where $V(\phi)$ is the potential, $X$ is the kinetic term, and $f(\phi)$ is the warp factor. For the IR DBI model, the inflaton potential is

$$
V(\phi)=V_{0}-\frac{1}{2} \beta H^{2} \phi^{2}
$$

where the parameter $\beta$ is in principle within a wide range $0.1<$ $\beta<10^{9}[11$ ].

The scalar power spectrum of DBI inflation can be parametrized as [11]

$$
\Delta_{\mathcal{R}}^{2}(k)=\frac{A_{s}}{N_{e}^{4}}\left(1-\frac{N_{c}^{16}}{N_{c}^{8}+\left(N_{e}^{\mathrm{DBI}}\right)^{8}}\right),
$$



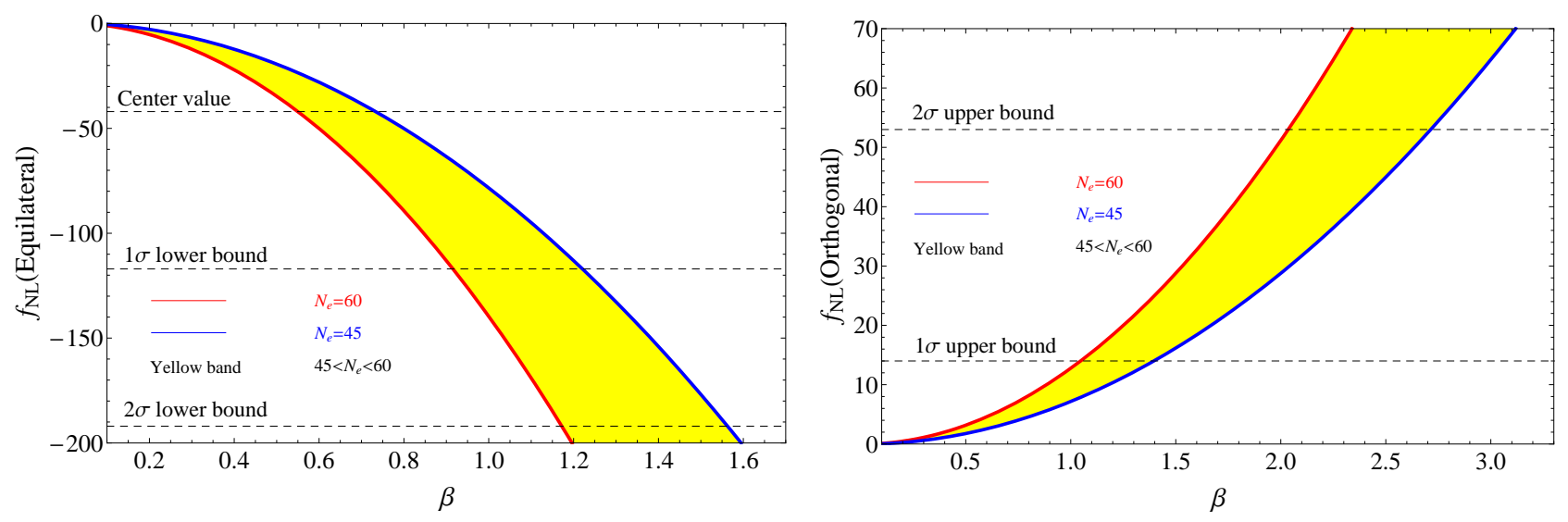

FIG. 6: Equilateral (left panel) and orthogonal (right panel) $f_{\mathrm{NL}}$ predictions for different $\beta$ values in the IR DBI model. The red and blue lines are for number of $e$-folds to be 60 and 45 . The yellow band is the allowed region given these two boundary of number of $e$-folds. We over-plot the best-fit values, $1 \sigma$ and $2 \sigma$ bounds for $f_{\mathrm{NL}}^{\mathrm{eq}}$ and $f_{\mathrm{NL}}^{\text {orth }}$ as dashed straight lines.

where $A_{s}$ is the amplitude of the perturbations which depends on several parameters of the internal space, $N_{c}$ is the critical number of $e$-folds at scale $k_{c}$ (critical scale where string phase transition happens), and $N_{e}^{\mathrm{DBI}}$ is the number of $e$-folds of inflation at relativistic rolling. The total number of $e$-folds is the sum of relativistic and nonrelativistic (NR) rollings,

$$
N_{e}^{\mathrm{tot}}=N_{e}^{\mathrm{DBI}}+N_{e}^{\mathrm{NR}}
$$

Now we can calculate the spectral index and its running, which turns out to be (see also Appendix in [11])

$$
\begin{aligned}
n_{s}-1 & =\frac{d \ln \Delta_{\mathcal{R}}^{2}(k)}{d \ln k} \\
& =\frac{4}{N_{e}^{\mathrm{DBI}}} \frac{x^{2}+3 x-2}{(x+1)(x+2)}, \\
\alpha_{s} & =\frac{d n_{s}}{d \ln k} \\
& =\frac{4}{\left(N_{e}^{\mathrm{DBI}}\right)^{2}} \frac{x^{4}+6 x^{3}-55 x^{2}-96 x-4}{(x+1)^{2}(x+2)^{2}},
\end{aligned}
$$

where $x=\left(N_{e}^{\mathrm{DBI}} / N_{c}\right)^{8}$.

In addition, nontrivial sound speed $c_{s}$ can generate large non-Gaussianity since the inflaton is no longer slowly rolling down to the potential. The predicted equilateral and orthogonal non-Gaussianities are [43, 44]

$$
\begin{aligned}
& f_{\mathrm{NL}}^{\mathrm{eq}}=-0.35 \frac{1-c_{s}^{2}}{c_{s}^{2}}, \\
& f_{\mathrm{NL}}^{\mathrm{orth}}=0.032 \frac{1-c_{s}^{2}}{c_{s}^{2}},
\end{aligned}
$$

where

$$
\frac{1}{c_{s}} \simeq \frac{\beta N_{e}^{\mathrm{DBI}}}{3}
$$

\section{B. Confront with current data}

Since the IR DBI model has a lot of parameters that describe the internal structure of the warped space, in order to directly compare its predictions with the current observational data, we adopt the best-fit values of $N_{c}, k_{c}$ and $N_{e}^{\mathrm{NR}}$ to be 35.7, $10^{-4.15} \mathrm{Mpc}^{-1}$ and 18.4 , respectively, according to the constraints from WMAP 5-year data [11].

In Fig. 5 we plot the predicted trajectory of the IR DBI model in the $\alpha_{s}-n_{s}$ plane. The purple and orange contours are the results from WMAP9+ and Planck+WP+BAO as we discussed before. The red line is the trajectory corresponding to $N_{e}^{\text {tot }}$ between 45 and 60, which includes a wide range of scale $k$. One can see that the trajectory crosses the contours of both WMAP9+ and Planck+WP+BAO, which is quite consistent with the data. In addition, the model predictions at the two pivot scales $k_{0}=0.002 \mathrm{Mpc}^{-1}$ and $k_{0}=0.038 \mathrm{Mpc}^{-1}$, which are the chosen scales of the two constraints are marked on the plot. One can see that the black dot is close to the boundary of WMAP9+ constraints while the blue one is outside of the $2 \sigma$ contours from Planck. However, although it seems that there is a discrepancy, we remind the reader that there is some uncertainty of the subsequent evolution after inflation, so it is reasonable to allow a broader range of number of $e$-folds for a given pivot scale.

Non-Gaussianity becomes an important tool to constrain such a non-slow-roll inflation model. The local, equilateral and orthogonal $f_{\mathrm{NL}}$ parameters are given by Eq. (7), which still do not show strong signal for non-Gaussianity. However, the error-bars of local, equilateral (and orthogonal) $f_{\mathrm{NL}}$ become a factor of two and four smaller than WMAP 9-year data [45]. Since the IR DBI model predicts vanishing local $f_{\mathrm{NL}}$, it is already consistent with the value given by Planck. Now we investigate the predictions of equilateral and orthogonal types of non-Gaussianity.

In Fig. 6, we plot the model predictions of $f_{\mathrm{NL}}^{\mathrm{eq}}$ and $f_{\mathrm{NL}}^{\text {orth }}$ and the current lower and upper bounds. The yellow bands in both panels are the allowed region for $N_{e}^{\text {tot }}$ in between 45 
and 60. Note that in Planck paper XXII [4], $N_{e}$ is just allowed to be $60-90$ when considering the constraints on the IR DBI model, while here we consider a more reasonable range of the number of $e$-folds. Given the yellow bands and the $2 \sigma$ lower bound for equilateral type of non-Gaussianity, we find that the value of $\beta$ needs to be smaller than 1.5 in order to prevent very negative equilateral non-Gaussianity. Similarly, on the right panel, we show that $\beta$ needs to be less than 2.5 in order to prevent large positive non-Gaussianity. These limits are consistent with the range of $\beta<0.7$ as found by Planck paper XXIV [5], which uses global likelihood analysis to obtain the limit. We should notice that this is already a fine-tuning for IR DBI model, because in this model $\beta$ has the lower limit $0.1(\beta<0.01$ is KKLMMT model as discussed in Sec. IV] but no real upper limit. Therefore, the current data is able to shrink the parameter space to be $[0.1, O(1)]$ is already a tight limit. Our comparison gives a intuitive understanding of why the parameter $\beta$ needs to be smaller than a certain value.

\section{CONCLUSION}

In this paper, we studied brane inflation with the Planck data and the joint data set from WMAP 9-year data, SPT, ACT, BAO and $H_{0}$ data. We first discussed the relationship between the number of $e$-folds and the corresponding pivot scale. We clarified the case where adopting different pivot scales of the constraints, the corresponding number of $e$-folds could be slightly different.

We then considered a toy model (prototype) of brane inflation where the problem of dynamic stabilization is neglected. Furthermore, we considered a more realistic "slow-roll" brane inflation model (namely, the KKLMMT model) and the DBI inflation model, and examined them with the Planck and WMAP9+ results.

For the toy model, we showed that the model is consistent with the observational data at $2 \sigma \mathrm{CL}$, given the fact that it prefers a red tilt close to 0.96 and a slightly negative running. For a comparison, in our previous work [20], we found that this type of brane inflation model is consistent with the WMAP 5 -year data at the level of $1 \sigma$. The situation does not change very much when we confront the model with WMAP9+ data and Planck data.

For the KKLMMT model, we first discussed how the model parameter $\beta$ affects its predictions of scalar power spectrum. Then we compared the model to the WMAP9+ data and Planck data. We found that in order for the model to provide the $\alpha_{s}$ and $n_{s}$ allowed by the tight constraints from Planck and $W M A P 9+$, the $\beta$ parameter needs to be fine-tuned to be less than $10^{-3}$. For comparison, by using the WMAP 3-year data in [23], we found that the KKLMMT model cannot fit $W M A P 3+$ SDSS data at the level of $1 \sigma$ and a fine-tuning, at least eight parts in a thousand, is needed at the level of $2 \sigma$. When the WMAP 5-year data becomes available, we found that the value of the parameter $\beta$ is constrained to be less than $O\left(10^{-2}\right)$ at the level of $2 \sigma[20]$. Thus, we can see that the problem of fine-tuning of $\beta$ becomes more severe when confronting with the recent observational data. Undoubtedly, this is not good news for the KKLMMT model.

Finally, we briefly discussed the current constraints on the infrared Dirac-Born-Infeld inflation model given the current observational data. The model can predict a larger negative running $(\sim-0.02)$ than the previous KKLMMT model. By figuring out the trajectory of the model on the $\alpha_{s}-n_{s}$ plane by varying the number of $e$-folds, we found that the model can predict the running of the spectral index and the tilt that are consistent with WMAP9+ and Planck data. However, when we confronted it with the current bounds on equilateral and orthogonal non-Gaussianities, we found that in order to avoid a large non-Gaussianity the value of $\beta$ which controls the shape of the potential needs to be less than 1.5. This limit to the IR DBI model is already a fine-tuning.

To summarize, although the prototype of brane inflation can fit the data well, it is not a realistic model of the brane inflation. For the KKLMMT and IR DBI inflation models, the parameters need to be fine-tuned to satisfy the current observational requirement. The current observation of CMB from Planck is competent to place stringent limits on internal parameters of warped space.

\section{Acknowledgments}

We would like to thank Anthony Challinor, Xingang Chen, Gary Hinshaw and Andrew Liddle for useful discussions. Y.Z.M. is supported by a CITA National Fellowship. Part of the research is supported by the Natural Science and Engineering Research Council of Canada. Q.G.H. is supported by the Knowledge Innovation Program of the Chinese Academy of Science and by the National Natural Science Foundation of China (Grant No. 10821504). X.Z. is supported by the National Natural Science Foundation of China (Grants No. 10705041, No. 10975032 and No. 11175042) and by the National Ministry of Education of China (Grants No. NCET09-0276, No. N100505001 and No. N120505003).
[1] G. Hinshaw et al., 2012, arXiv:1212.5226

[2] P.A.P. Ade et al., Planck 2013 results. I, arXiv: 1303.5062 [astro-ph].

[3] P.A.P. Ade et al., Planck 2013 results. XVI, arXiv: 1303.5076 [astro-ph].

[4] P.A.P. Ade et al., Planck 2013 results. XXII, arXiv: 1303.5082 [astro-ph].
[5] P.A.P. Ade et al., Planck 2013 Results. XXIV, arXiv:1303.5084 [astro-ph].

[6] L. Anderson et al., MNRAS, 427, 3435 (2012).

[7] J. Sievers et al., 2013, arXiv: 1301.0824

[8] Z. Hou et al., 1212, arXiv: 1212.6267

[9] A. H. Guth, Phys. Rev. D 23, 347 (1981).

[10] A. D. Linde, Phys. Lett. B 108, 389 (1982). 
[11] R. Bean, X. Chen, H. Peiris \& J. Xu, Phys. Rev. D 77, 023527 (2008).

[12] A. A. Starobinsky, Phys. Lett. B 91, 99 (1980).

[13] J. Martin \& R. H. Brandenberger, Phys. Rev. D 63, 123501 (2001).

[14] G. R. Dvali \& S. H. H. Tye, Phys. Lett. B 450, 72 (1999).

[15] S. H. H. Tye, Lect. Notes Phys. 737, 949 (2008), arXiv:hep-th/0610221.

[16] C. P. Burgess, M. Majumdar, D. Nolte, F. Quevedo, G. Rajesh and R. J. Zhang, JHEP 07, (2001) 047, arXiv:hep-th/0105204.

[17] F. Quevedo, Class. Quant. Grav. 195721 (2002), [hep-th/0210292].

[18] G. R. Dvali, Q. Shafi \& S. Solganik, arXiv:hep-th/0105203

[19] S. Kachru, R. Kallosh, A. Linde, J. M. Maldacena, L. McAllister and S. P. Trivedi, JCAP 10, 013 (2003), hep-th/0308055

[20] Y. Z. Ma \& X. Zhang, JCAP 03, 006 (2009).

[21] H. Firouzjahi \& S.H. Tye, JCAP 03, 009 (2005).

[22] D. Baumann, \& L. McAllister, hep-th/0610285.

[23] Q. G. Huang, M. Li and J. H. She, JCAP 11 (2006) 010 [hep-th/0604186].

[24] Q. G. Huang, Phys. Rev. D 74, 063513 (2006) [arXiv:astro-ph/0605442].

[25] X. Zhang, JCAP 12 (2006) 002 [hep-th/0608207|.

[26] D. Baumann, A. Dymarsky, I. R. Klebanov \& L. McAllister, JCAP 01, 024, (2008).

[27] D. Baumann, A. Dymarsky, S. Kachru, I. R. Klebanov \& L. McAllister, JHEP, 06, 072 (2010).

[28] E. Silverstein \& D. Tong, Phys. Rev. D 70, 103505 (2004).
[29] M. Alishahiha, E. Silverstein \& D. Tong, Phys. Rev. D 70, 123505 (2004).

[30] X. Chen, Phys. Rev. D 71, 063506 (2005).

[31] X. Chen, JHEP 08, 045 (2005).

[32] X. Chen, Phys. Rev. D 72, 123518 (2005).

[33] F. Beulter et al., MNRAS, 416, 3017 (2011).

[34] C. Blake et al., MNRAS, 425, 405 (2012).

[35] A. G. Riess et al. ApJ, 730, 119 (2011).

[36] C. Cheng, Q.G. Huang \& Y.Z.Ma, arXiv: 1303.4497 [astroph.CO].

[37] A. R. Liddle \& S. M. Leach, Phys. Rev. D 68, 103503 (2003).

[38] G. Efstathiou \& S. Gratton, JCAP 06, 011 (2009).

[39] CMBPol Study Team collaboration, D. Baumann et al., CMBPol mission concept study: probing inflation with CMB polarization, AIP Conf. Proc. 1141, 10 (2009), arXiv:0811.3919].

[40] Y.Z.Ma, W. Zhao \& M. Brown, JCAP 10, 007 (2010).

[41] A. Liddle \& D. H. Lyth, Cosmological Inflation and Large Scale Structure, Cambridge University Press, 2000.

[42] C. Cheng, Q. G. Huang, X. D. Li \& Y. Z. Ma, Phys. Rev. D 86, 123512 (2012).

[43] X. Chen, M. X. Huang, S. Kachru \& G. Shiu, JCAP 01, 002 (2007).

[44] L. Senatore, K. M. Smith \& M. Zaldarriaga, JCAP 01, 028 (2010).

[45] C. L. Bennett et al., arXiv: 1212.5225 [astro-ph]. 